\title{
Luteinizing hormone facilitates angiogenesis in ovarian epithelial tumor cells and metformin inhibits the effect through the mTOR signaling pathway
}

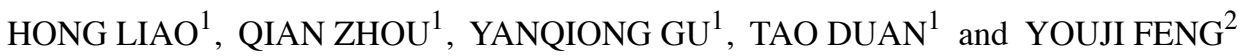 \\ ${ }^{1}$ Clinical and Translational Research Center, Shanghai First Maternity and Infant Hospital, \\ Tongji University School of Medicine; ${ }^{2}$ Department of Obstetrics and Gynecology, \\ Shanghai Jiao Tong University Affiliated First People's Hospital, Shanghai, P.R. China
}

Received December 12, 2011; Accepted February 17, 2012

DOI: $10.3892 /$ or.2012.1745

\begin{abstract}
High levels of gonadotropin are a risk factor for ovarian cancer development. Aberrant gonadotropin levels benefit tumor angiogenesis, but the detailed mechanism is not clear. Therefore, the aim of this study was to investigate the molecular mechanism of high levels of luteinizing hormone (LH) on the promotion of tumor angiogenesis and to outline a feasible therapeutic strategy. Western blotting and immunofluorescence staining were used to determine the effect of LH on VEGF and Slit2 expression and examine the signaling pathway involved in regulating the expression of both molecules. Real-time PCR was used to investigate the effect of metformin on LH induction of VEGF and Slit2 expression. It was found that $50 \mathrm{mIU} / \mathrm{ml}$ LH significantly upregulated VEGF and Slit2 expression, and activated the PI3K/AKT-mTOR signaling pathway. However, metformin inhibited the mTOR signaling pathway and further blocked LH-induced VEGF and Slit2 expression. In conclusion, high levels of LH promote angiogenesis in ovarian cancer via the PI3K/AKT-mTOR pathway. However, metformin could inhibit tumor angiogenesis by blocking the mTOR signaling pathway.
\end{abstract}

\section{Introduction}

Luteinizing hormone ( $\mathrm{LH})$, one of the gonadotropins, is a pituitary glycoprotein hormone that regulates ovarian steroido-

Correspondence to: Professor Tao Duan, Department of Obstetrics and Gynecology, Shanghai First Maternity and Infant Hospital, Tongji University School of Medicine, 536 Changle Road, Shanghai 200080, P.R. China

E-mail: tduan@yahoo.com

Professor Youji Feng, Department of Obstetrics and Gynecology, Shanghai First People's Hospital, Shanghai Jiao Tong University, 100 Haining Road, Shanghai 200080, P.R. China

E-mail: fengyj4806@sohu.com

Abbreviations: LH, luteinizing hormone; FSH, follicle-stimulating hormone; OEC, ovarian epithelial cancer

Key words: luteinizing hormone, angiogenesis, metformin, vascular endothelial growth factor, Slit2 genesis. The gonadotropin theory of ovarian cancer proposes that accumulated serum gonadotropins, follicle-stimulating hormone (FSH) and LH, contribute to the development of ovarian cancer. A growing body of evidence has implicated the role of $\mathrm{LH}$ in the etiology of ovarian epithelial cancer (OEC). Previous studies show that LH inhibits Fas-induced apoptosis in ovarian cancer and supports the hypothesis of hormonal involvement in ovarian carcinogenesis (1). In addition, it reports that LH could enhance the invasion and migration of ovarian cancer via the PI3K/AKT signaling pathway. Recent evidence suggests that gonadotropins promote ovarian cancer OV207 and OVCAR-3 cell migration and proliferation by activation of ERK1/2 signaling in a calciumand PKC $\delta$-dependent manner (2). However, little is known about the role of $\mathrm{LH}$ in tumor angiogenesis.

Vascular endothelial growth factor (VEGF) is a glycoprotein which is associated with tumor angiogenesis (3). Higher levels of VEGF are consistent with poor prognosis and tumor progression in various types of tumors, including ovarian cancer (4). Elevated levels of gonadotropin are risk factors for ovarian cancer and have multiple bio-activations; a study addressed the role of LH on angiogenesis in ovarian cancer. Wang et al reported that LH induced VEGF expression in AO cells in a dose-dependent manner. This study suggests that VEGF may accelerate ovarian tumor growth and tumor recurrence under elevated gonadotropin levels after menopause or surgery (5). However, the detailed mechanism of the LH-induced VEGF expression is not clear. Except of VEGF, a recent study indicates that Slit2, a secreted protein known to function through the Roundabout (Robo) receptor as a chemorepellent in axon guidance and neuronal migration, may also be involved in tumor angiogenesis. This study suggests that Slit2 induces tumor angiogenesis by Slit-Robo signaling; blocking this signaling could effectively inhibit cellular growth (6).

However, information has been obtained concerning the pathway triggering angiogenesis in ovarian cancer under elevated levels of gonadotropin, especially in a post-menopause state. Previous studies suggest that FSH, one of the gonadotropins, enhances HIF-1 activity downstream of the PI3-kinase/ AKT/Rheb/mTOR pathway and further induces VEGF expression (7). However, whether this signaling pathway is also involved in LH-induced angiogenesis is not clear. 
Table I. Primers used to amplify the target genes.

\begin{tabular}{llr}
\hline Gene & \multicolumn{1}{c}{ Sequence } & Annealing temperature $\left({ }^{\circ} \mathrm{C}\right)$ \\
\hline VEGF & Forward : 5'-CCT TGC TGC TCT ACC TCC AC-3' & 60 \\
& Reverse : 5'-ATC TGC ATG GTG ATG TTG GA-3' & 60 \\
Slit2 & Forward: 5'-TCA GCT GTT TCC TGA GTT GC-3' & 60 \\
GAPDH & Reverse: 5'-TGG TTG AAA CTT GCC ACA GA-3' & 53 \\
& Forward: 5'-AAC GGA TTT GGT CGT ATTG-3' & \\
\hline
\end{tabular}

Although a recent study confirmed that metfomin inhibited the proliferation of various type of cancer cells mainly by activating AMPK and further repressing mTOR (8-10), it is still remains unknown whether metformin could block or attenuate LH-induced angiogenesis. Therefore, the specific interest of the present study was to examine the role of $\mathrm{LH}$ on VEGF and Slit2 expression and to define its detailed molecular mechanism of action.

\section{Materials and methods}

Ovarian cancer cell lines and cell culture. The ovarian cancer cell line Hey was maintained in our laboratory and cultured in DMEM/F12 supplemented with $10 \%$ fetal bovine serum (FBS), $100 \mathrm{U} / \mathrm{ml}$ penicillin, $100 \mu \mathrm{g} / \mathrm{ml}$ streptomycin, sodium pyruvate, and L-glutamine in humidified $5 \% \mathrm{CO}_{2}$ at $37^{\circ} \mathrm{C}$. Another cell line MCV152 was derived from SV-40transformed benign ovarian serous papillary cystadenoma and transfected with telomerase hTERT. The culture conditions were previously described (11).

Western blot analysis. To investigate the effect of LH on VEGF and Slit2 expressions, western blot analysis was performed. Briefly, different dose of LH were used to treat MCV152 and Hey cells for $48 \mathrm{~h}$, and the cells were collected for use. In order to determine whether LH regulated VEGF and Slit2 by the PI3K/AKT/mTOR signaling pathway, $50 \mathrm{mIU} / \mathrm{ml} \mathrm{LH}$ was used to treat Hey cells for various times, and the cells were then collected. Subsequently, the Hey cells were pre-treated with $100 \mathrm{nM}$ rapamycin for $1 \mathrm{~h}, 50 \mathrm{mIU} / \mathrm{ml} \mathrm{LH}$ stimulated for another $48 \mathrm{~h}$, and the treated Hey cells were collected for use. The following steps performed have been described in a previous report (11). Briefly, after lysis, $60 \mu \mathrm{g}$ proteins were loaded on $10 \%$ SDS-PAGE gels, transferred to polyvinylidene fluoride (PVDF) membranes, and blocked in 5\% non-fat milk in $10 \mathrm{mM}$ Tris, $\mathrm{pH} 7.5,100 \mathrm{mM} \mathrm{NaCl}$, and $0.1 \%$ (w/v) Tween-20 for $2 \mathrm{~h}$. Anti-human VEGF, anti-Slit2, antiphosphorylated AKT, anti-AKT, anti-phosphorylated mTOR, anti-mTOR, anti- $\beta$-actin, and anti-GAPDH primary antibodies were diluted 1:1000 and incubated overnight, followed by 1-h incubation with the appropriate secondary antibody at room temperature. The protein bands were visualized with the ECL Plus system (Amersham, GE Healthcare).

Immunocytochemistry assay. MCV152 and Hey cells were plated in 6-well plates with coverslips for $24 \mathrm{~h}$. After an addi- tional 24-h serum-free starvation, the cells were treated with $50 \mathrm{mIU} / \mathrm{ml} \mathrm{LH}$ for another $48 \mathrm{~h}$. Subsequently, the cells were washed with PBS three times, fixed for 10 min with formaldehyde and then permeabilized in $0.1 \%$ Triton X-100 in PBS for 5 min. The anti-human VEGF and Slit2 primary antibodies were diluted in 1:100 and incubated at $37^{\circ} \mathrm{C}$ for $1 \mathrm{~h}$. Following this, the cultures were rinsed three times in PBS for 5 min and incubated with anti-mouse or anti-rabbit IgG FITC-conjugated secondary antibodies for $45 \mathrm{~min}$ at room temperature and then washed three times with PBS. Hoechst 33258 was used to stain the nucleus for $10 \mathrm{sec}$. After the PBS wash, the inverted fluorescence microscope was used to observe the expression of VEGF and Slit2.

Hormone treatment and metformin stimulation. Hey cells were seeded in $10-\mathrm{cm}$ dishes at a concentration of $1 \times 10^{5} / \mathrm{ml}$. After serum-free starvation for $24 \mathrm{~h}$, cells were treated with various doses of metformin $(0.01,0.1$ and $1 \mathrm{mM})$ for another $48 \mathrm{~h}$, and the proteins were collected to determine the effect of metformin on VEGF and Slit2 expression by western blot analysis. To investigate whether metformin could attenuate LH-induced VEGF and Slit2 expression, Hey cells were treated alone with metformin or LH or treated with both reagents in combination for $48 \mathrm{~h}$. The cells were collected to study whether metformin blocked the effect of LH on VEGF and Slit 2 expression.

RNA extraction, RT-PCR and real-time PCR. The RNA extraction, RT-PCR and real-time PCR reactions were performed as described in a previous report (11). Briefly, MCV152 and Hey cells were plated in 6-well plate and cultured for $24 \mathrm{~h}$. The medium was changed with opti-MEM and cultured for another $24 \mathrm{~h}$. The cells were treated alone with LH, rapamycin, metformin, or treated with $\mathrm{LH}$ and rapamycin, or $\mathrm{LH}$ and metformin in combination for $24 \mathrm{~h}$, and collected. Total RNA was isolated according to the protocol of the RNeasy micro kit (Qiagen, Frankfurt, Germany). RNA ( $2 \mu \mathrm{g})$ was reverse transcribed into cDNA in a $20 \mu \mathrm{l}$ reaction according to the protocol of teh PrimeScriptT ${ }^{\mathrm{TM}}$ RT reagent kit (Takara, Shuzo, Kyoto, Japan). The primers are listed in Table I. The reactions were set up with $10 \mu \mathrm{l}$ SYBR-Green PCR Master Mix (Takara), $1.0 \mu 110$ umol primer mixture, and $2 \mu \mathrm{l}$ cDNA template. The real-time PCR was performed following the conditions: $95^{\circ} \mathrm{C}$ for $15 \mathrm{sec}$ followed by 35 cycles of $60^{\circ} \mathrm{C}$ and $72^{\circ} \mathrm{C}$ for $30 \mathrm{sec}$. Relative mRNA levels of target genes were normalized to GAPDH. 
A

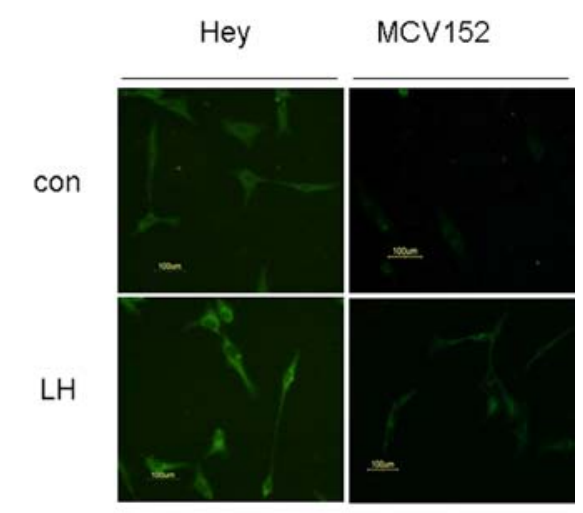

B

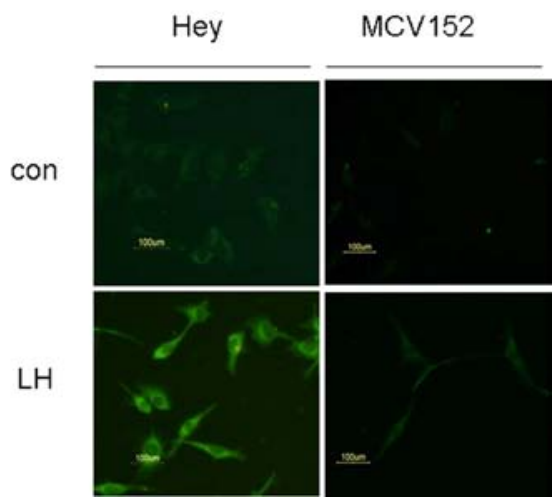

Figure 1. LH induces VEGF and Slit2 expressions in ovarian cancer. LH-induced Slit2 (A) and VEGF (B) expression were detected by immunocytochemistry.

Data analysis. The band density of western blot analsyis and relative target gene mRNA levels were analyzed with one-way ANOVA. P-values of $<0.05$ were considered statistically significant.

\section{Results}

LH upregulates VEGF and Slit2 expressions. Both VEGF and Slit2 are important factors in tumor angiogenesis. In order to determine the effect of LH on the expression of both factors, an immunofluorescence assay and western blot analysis were performed. As shown in Fig. 1, stimulation with $50 \mathrm{mIU} / \mathrm{ml} \mathrm{LH}$ resulted in clear upregulation of Slit2 and VEGF expression in MCV152 or Hey cells. The green fluorescence represented the expression of VEGF or Slit2, compared with the non-treatment control, the significantly enhanced fluorescence could be observed with LH treatment in both cell lines. Western blot analysis demonstrated the dose-dependent effects of LH stimulation on VEGF and Slit2 expression. Maximal induction of VEGF by LH was obtained at a concentration of $50 \mathrm{mIU} / \mathrm{ml}$ in Hey cells and of $400 \mathrm{mIU} / \mathrm{ml}$ in MCV152 cells. Similar expression pattern was observed for Slit2. The most significant induction occurred at a dose of $100 \mathrm{mIU} / \mathrm{ml}$ with a 3.2 -fold increase in Hey cells and at $50 \mathrm{mIU} / \mathrm{ml}$ in MCV152 cells with a 3.5-fold increase (Fig. 2).

LH activates the PI3K/AKT-mTOR signaling pathway. In order to gain insight into the mechanism of LH-induced angio-
A

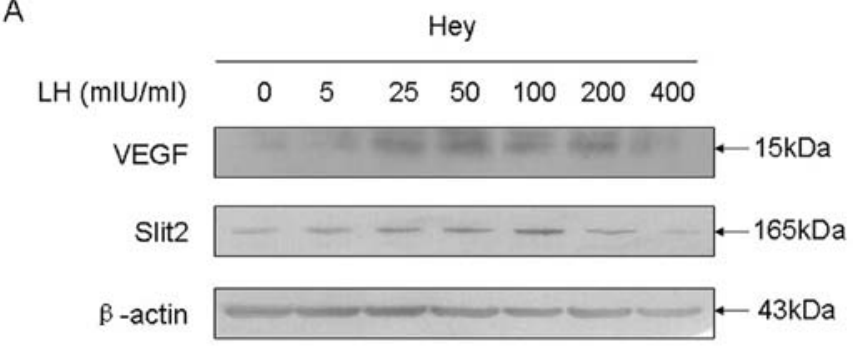

B

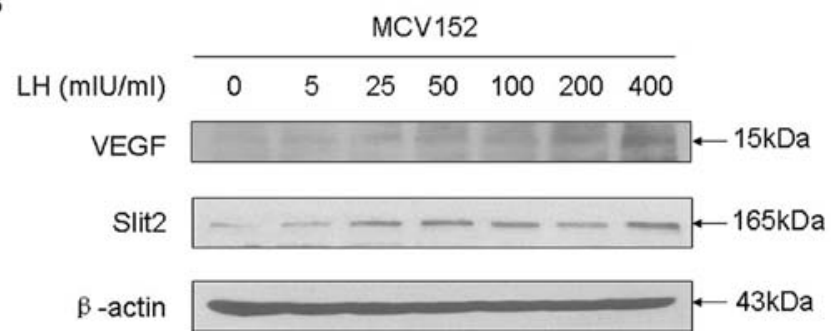

Figure 2. Dose-response of LH-induced VEGF and Slit2 protein levels in ovarian cancer. The effects of LH on VEGF and Slit2 expression in (A) Hey cells and (B) in MCV152 cells were determined by western blotting. In A and $\mathrm{B}$, the upper panel shows the protein level of VEGF after treatment with a series of concentrations of LH for $48 \mathrm{~h}$, the middle panel presents the blot of Slit2, while the lower panel shows $\beta$-actin protein level. $\beta$-actin was used as a loading control.

genesis, western blot analysis was performed to investigate the $\mathrm{PI} 3 \mathrm{~K} / \mathrm{AKT}$-mTOR signaling pathway. $\mathrm{LH}(50 \mathrm{mIU} / \mathrm{ml})$ induced the phosphorylation of AKT in a time-dependent fashion in Hey cells (Fig. 3). The maximal induction of AKT phosphorylation occurred at $45 \mathrm{~min}$. Activation of mTOR also showed a similar expression pattern (Fig. 3). LH treatment resulted in a clear increase of mTOR phosphorylation, which mediates downstream targeting genes. A peak level of phosphorylation of mTOR occurred at 30 min with LH stimulation.

Rapamycin attenuates LH-induced VEGF and Slit 2 expression. Since the mTOR signaling pathway is involved in $\mathrm{LH}$-induced angiogenesis, we investigated the effect of blocking this pathway on VEGF and Slit2 expression. The Western blot assay indicated that rapamycin could downregulate both VEGF and Slit2 expressions. Furthermore, LH plus rapamycin treatment did not maintain LH-induced VEGF and Slit2 protein levels; this regulation was inhibited by rapamycin through inhibiting the mTOR signaling pathway (Fig. 4).

Metformin inhibits LH-induced angiogenesis by blocking the mTOR signaling pathway. The above study confirmed that the mTOR signaling pathway was involved in LH-induced angiogenesis. Therefore, targeting this pathway may be an effective therapeutic strategy. A recent study suggests that metformin could lower cancer risk, reduce the rate of cancer deaths $(12,13)$, and kill cancer cells. These effects were mainly dependent on targeting the AMPK-mTOR signaling pathway $(10,14)$. In the present study, the results showed that metformin treatment resulted in downregulation of VEGF and Slit2 in a dose-dependent manner. The maximum reduction was induced by $1 \mathrm{mM}$ metformin with an 8.4-fold decrease of VEGF and 

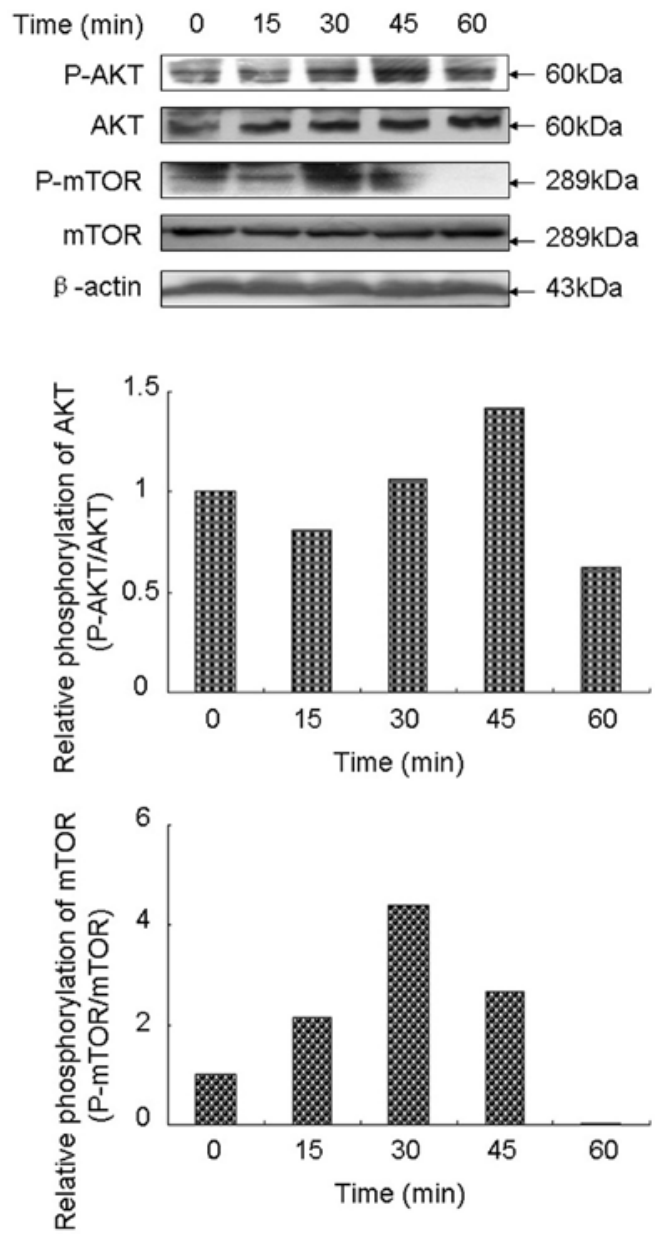

Figure 3. Western blot analyses showing LH-dependent phosphorylation of AKT and mTOR in Hey cells. Hey cells were treated with $50 \mathrm{mIU} / \mathrm{ml} \mathrm{LH}$ for the indicated time periods in serum-free cultures. The harvested cells were lysed and western blot analysis were performed as described in Materials and methods. $\beta$-actin was used as a loading control. PBS treatment served as a negative control.

a 2.2-fold decrease of Slit2 (Fig. 5A). Moreover, combination treatment with $\mathrm{LH}$ and metformin resulted in a reduction of LH-induced phosphorylation of mTOR, VEGF and Slit2 in Hey cells (Fig. 5B). To investigate whether similar patterns exist at the mRNA level, real-time PCR was performed to determine the mRNA levels of VEGF and Slit2. Similarly to rapamycin, metformin significantly inhibited the enhanced effect of LH on VEGF and Slit2 mRNA levels (Fig. 6).

\section{Discussion}

Increasing evidence suggests that the hormone environment is an important factor for cancer development, especially in ovarian cancer $(8,11,14-16)$. These hormones, including gonadotropin, gonadotropin releasing hormone and progestin, often play multiple roles; they commonly regulate cellular growth, cell migration and apoptosis. Moreover, in gynecological cancers, gonadotropin induces angiogenesis which is an important step in cancer development, growth, and invasion beyond regional borders (5). In this process, VEGF plays an important role whose expression often consists of tumor development and metastasis. Several studies addressed the
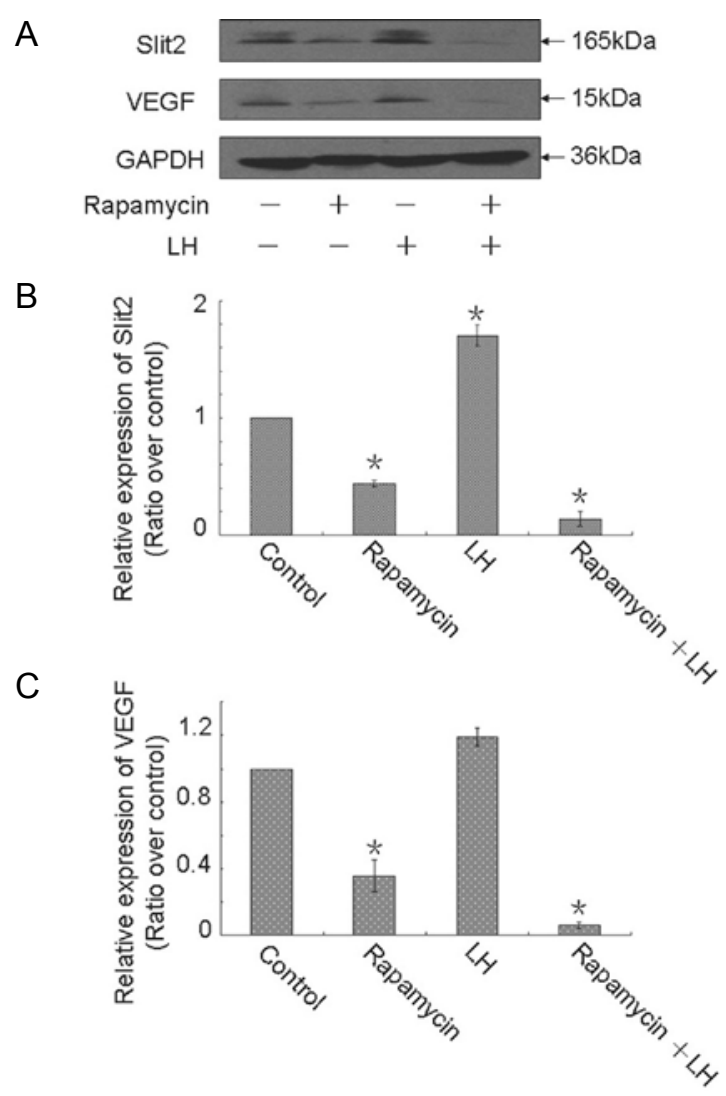

Figure 4. The specific inhibitor mTOR attenuates LH-induced VEGF and Slit2 expression. (A) The upper panel shows the protein level of Slit2 level after treatment with indicated reagents; the middle panel is a representative blot of VEGF; the lower panel shows the protein level of GAPDH which serves as a loading control. Relative expression of (B) Slit2 and (C) VEGF.
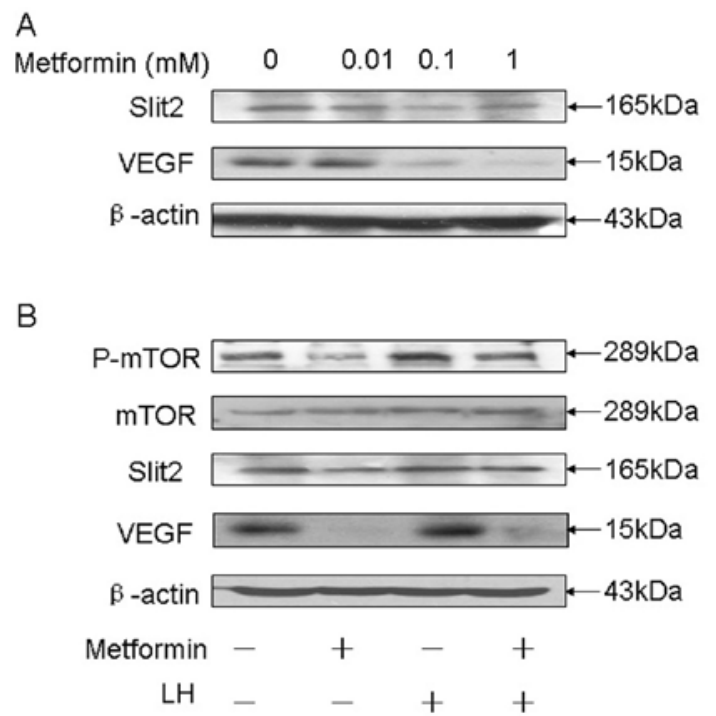

Figure 5. Metformin blocks PI3K/AKT-mTOR and abolishes LH-induced VEGF and Slit2 expressions. (A) Dose-response of metformin-induced inhibition of VEGF and Slit2 expressions in Hey cells. (B) Metformin inhibits phosphorylation of mTOR and attenuates LH-induced phosphorylation and further reduces the expressions of LH-induced Slit2 and VEGF.

relationship between hormones and $\operatorname{VEGF}(17,18)$, but the detailed molecular mechanism is not clear. In the current study, we found that LH potently upregulated VEGF expression in a 

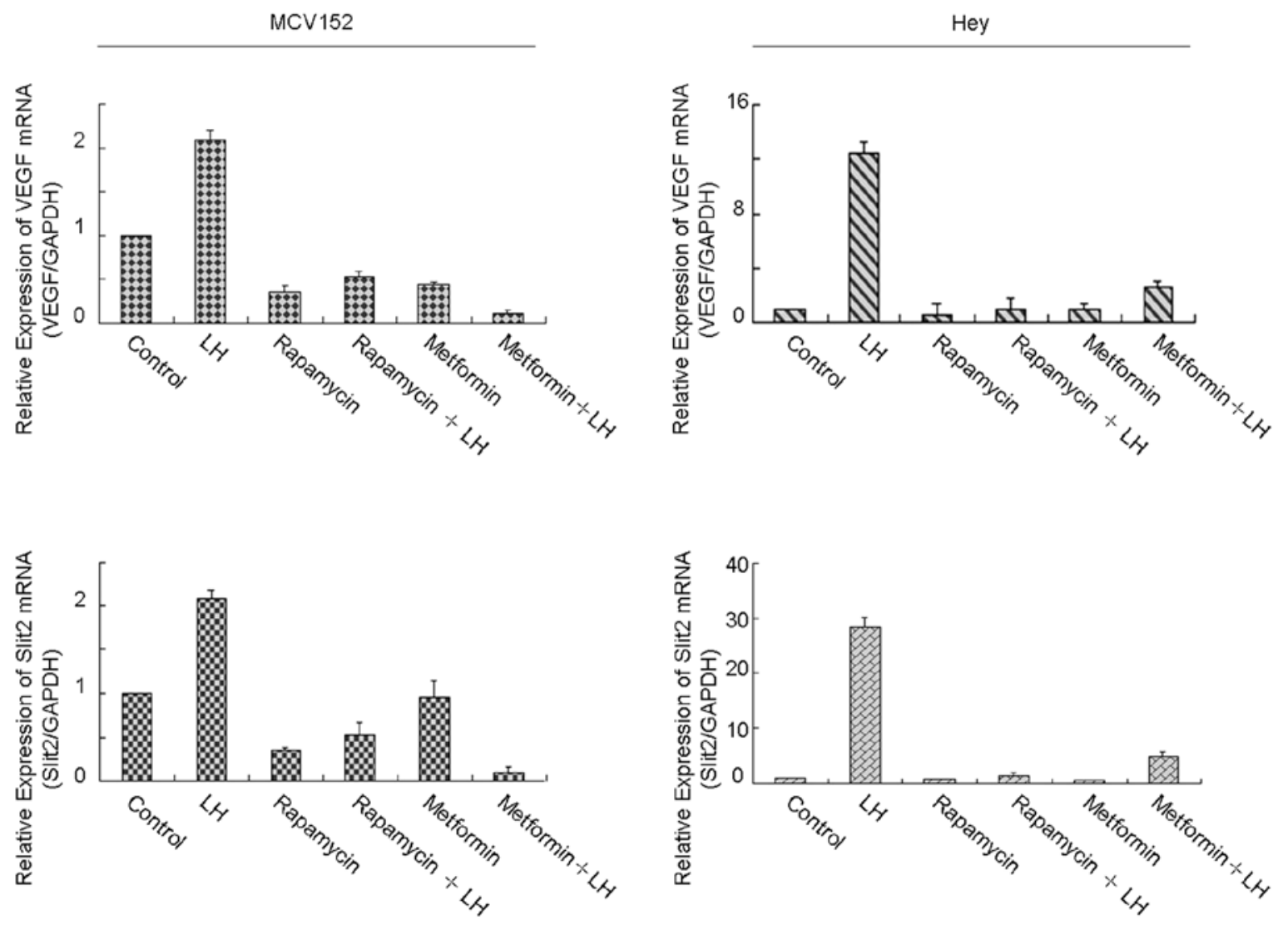

Figure 6. The effects of rapamycin and metformin on VEGF and Slit2 mRNA levels. Rapamycin (100 nM) and metformin (1 mM) abolishes LH-induced VEGF and Slit2 mRNA expressions in MCV152 cells (left column), and similar expression patters shows in Hey cells (right column). GAPDH is used as a loading control, relative mRNA levels of target genes are normalized to GAPDH.

dose-dependent manner (Figs. 1 and 2). This finding is consistent with the gonadotropin theory which states that it is the hormonal environment of the normal ovarian surface epithelium (OSE) and ovarian epithelial tumor (OET) cells that are associated with the development and progression of ovarian cancer. Exposure to excess gonadotropins has been proposed as a risk factor for ovarian cancer from the epidemiologic point of view (19), especially in postmenopausal women or in women who have received treatment for induction of ovulation $(20,21)$. The loss of the feedback suppression of estrogens, results in an increase in gonadotropin levels which are a risk factor for ovarian cancer. Thus, similarly to our in vitro experiment, the higher level of LH or FSH in vivo may enhance the expression of VEGF, and promote angiogenesis and finally enhance the blood supply to tumors.

The role of Slit-Roundabout (Robo) signaling in axon guidance is well-understood $(22,23)$. However, its role on angiogenesis has not been thoroughly investigated. A previous study showed that Slit 2 was expressed in a variety of human cancer cells where it acts as a chemoattractant to vascular endothelial cells to promote tumor angiogenesis. Recent research indicates that targeting Slit-Robo signaling may offer a novel anti-angiogenesis approach for various cancer therapies $(24,25)$. Here, we showed the effect of hormone on Slit2 expression. Similarly to the VEGF expression pattern, LH significantly and dose-dependently upregulated the Slit2 protein levels. Thus, the elevated gonadotropin levels after postmenopause or when undergoing oophorectomy may promote tumor growth and enhance the recurrence by upregulating VEGF and Slit2 which benefit cancer cell growth, shedding, migration, invasion and configuring of the neovasculature.

The signaling pathway of gonadotropin-induced migration and invasion has been well-described $(2,19)$; however, little information is available about the signaling of LH-induced angiogenesis. In the present study, we observed that $\mathrm{LH}$ could activate AKT and mTOR in a time-related manner. The following experiment indicated that rapamycin, the specific inhibitor of mTOR, potently repressed VEGF and Slit2 expressions and attenuated LH-induced VEGF and Slit2 protein and mRNA levels (Figs. 4 and 6). This result suggested that the PI3K/AKT-mTOR pathway was a major signal for triggering angiogenesis with LH treatment.

Since the mTOR signaling pathway is involved in LH-induced angiogenesis, targeting mTOR may be an effective therapeutic strategy for the treatment of ovarian cancer. In agreement with the results of a previous study, we also found that rapamycin significantly inhibited the mTOR signaling pathway and further mediated the expression of target genes downstream of mTOR. Recently, it was reported that metformin could inhibit proliferation of various types of cancer cells mainly by activating AMPK and further repressing mTOR (8-10). Therefore, we further investigated that effect of metformin on LH-induced VEGF and Slit2 expression. The current data showed that metfomin inhibited phosphorylation of mTOR and repressed VEGF and Slit2 expression in a doserelated manner. Moreover, metformin blocked the effect of 
LH on VEGF and Slit2 expression by inhibiting the mTOR pathway. Our study suggests that mTOR may be a critical targeting molecule for ovarian cancer therapy. In addition, metformin may be a potential antitumor agent because it abolished LH-induced activation of mTOR and thus may further inhibit the neovasculature.

Taken together, the current study provides novel insight to the clinical implications of hormonal intervention in ovarian cancer. In addition, metformin may be an adjunctive therapy after surgery or the early developmental stage of ovarian cancer due to its ability to decrease elevated LH-induced angiogenesis. However, their beneficial effects in clinical trials need to be further evaluated.

\section{Acknowledgements}

This study was supported in part by the National Natural Science Foundation of China (NSFC no. 30872755) fund to Dr Youji Feng, and supported by 200902 from the Shanghai First Mternity and the Infant hospital, Tongji University School of Medicine.

\section{References}

1. Slot KA, de Boer-Brouwer M, Houweling M, Vaandrager AB Dorrington JH and Teerds KJ: Luteinizing hormone inhibits Fas-induced apoptosis in ovarian surface epithelial cell lines. J Endocrinol 188: 227-239, 2006.

2. Mertens-Walker I, Bolitho C, Baxter RC and Marsh DJ: Gonadotropin-induced ovarian cancer cell migration and proliferation require extracellular signal-regulated kinase 1/2 activation regulated by calcium and protein kinase C $\delta$. Endocr Relat Cancer 17: 335-349, 2010.

3. Dvorak HF, Orenstein NS, Carvalho AC, et al: Induction of a fibrin-gel investment: an early event in line 10 hepatocarcinoma growth mediated by tumor-secreted products. J Immunol 122: 166-174, 1979

4. Wong C, Wellman TL and Lounsbury KM: VEGF and HIF-1 $\alpha$ expression are increased in advanced stages of epithelial ovarian cancer. Gynecol Oncol 91: 513-517, 2003.

5. Wang J, Luo F, Lu JJ, Chen PK, Liu P and Zheng W: VEGF expression and enhanced production by gonadotropins in ovarian epithelial tumors. Int J Cancer 97: 163-167, 2002.

6. Wang B, Xiao Y, Ding BB, et al: Induction of tumor angiogenesis by Slit-Robo signaling and inhibition of cancer growth by blocking Robo activity. Cancer Cell 4: 19-29, 2003.

7. Alam H, Maizels ET, Park Y, et al: Follicle-stimulating hormone activation of hypoxia-inducible factor-1 by the phosphatidylinositol 3-kinase/AKT/Ras homolog enriched in brain (Rheb)/ mammalian target of rapamycin (mTOR) pathway is necessary for induction of select protein markers of follicular differentiation. J Biol Chem 279: 19431-19440, 2004.

8. Cantrell LA, Zhou C, Mendivil A, Malloy KM, Gehrig PA and Bae-Jump VL: Metformin is a potent inhibitor of endometria cancer cell proliferation-implications for a novel treatment strategy. Gynecol Oncol 116: 92-98, 2009.
9. Hirsch HA, Iliopoulos D, Tsichlis PN and Struhl K: Metformin selectively targets cancer stem cells, and acts together with chemotherapy to block tumor growth and prolong remission. Cancer Res 69: 7507-7511, 2009.

10. Rattan R, Giri S, Hartmann LC and Shridhar V: Metformin attenuates ovarian cancer cell growth in an AMP-kinase dispensable manner. J Cell Mol Med 15: 166-178, 2011.

11. Zhang Z, Jia L, Feng Y and Zheng W: Overexpression of folliclestimulating hormone receptor facilitates the development of ovarian epithelial cancer. Cancer Lett 278: 56-64, 2009.

12. Jiralerspong S, Palla SL, Giordano SH, et al: Metformin and pathologic complete responses to neoadjuvant chemotherapy in diabetic patients with breast cancer. J Clin Oncol 27: 3297-3302, 2009.

13. Evans JM, Donnelly LA, Emslie-Smith AM, Alessi DR and Morris AD: Metformin and reduced risk of cancer in diabetic patients. BMJ 330: 1304-1305, 2005.

14. Morch LS, Lokkegaard E, Andreasen AH, Kruger-Kjaer S and Lidegaard O: Hormone therapy and ovarian cancer. JAMA 302: 298-305, 2009.

15. MacLennan AH and Baber R: Hormone therapy use and risk of ovarian cancer. JAMA 302: 2203-2204, 2009.

16. Hecht JL, Kotsopoulos J, Hankinson SE and Tworoger SS: Relationship between epidemiologic risk factors and hormone receptor expression in ovarian cancer: results from the Nurses' Health Study. Cancer Epidemiol Biomarkers Prev 18: 1624-1630, 2009.

17. Boocock CA, Charnock-Jones DS, Sharkey AM, et al: Expression of vascular endothelial growth factor and its receptors flt and KDR in ovarian carcinoma. J Natl Cancer Inst 87: 506-516, 1995.

18. Paley PJ, Staskus KA, Gebhard K, et al: Vascular endothelial growth factor expression in early stage ovarian carcinoma. Cancer 80: 98-106, 1997.

19. Choi JH, Choi KC, Auersperg N and Leung PC: Gonadotropins activate proteolysis and increase invasion through protein kinase $\mathrm{A}$ and phosphatidylinositol 3-kinase pathways in human epithelial ovarian cancer cells. Cancer Res 66: 3912-3920, 2006.

20. Venn A, Watson L, Bruinsma F, Giles G and Healy D: Risk of cancer after use of fertility drugs with in vitro fertilisation. Lancet 354: 1586-1590, 1999.

21. Brekelmans CT: Risk factors and risk reduction of breast and ovarian cancer. Curr Opin Obstet Gynecol 15: 63-68, 2003.

22. Seeger M, Tear G, Ferres-Marco D and Goodman CS: Mutations affecting growth cone guidance in Drosophila: genes necessary for guidance toward or away from the midline. Neuron 10: 409-426, 1993.

23. Kidd T, Brose K, Mitchell KJ, et al: Roundabout controls axon crossing of the CNS midline and defines a novel subfamily of evolutionarily conserved guidance receptors. Cell 92: 205-215, 1998.

24. Wang LJ, Zhao Y, Han B, et al: Targeting Slit-Roundabout signaling inhibits tumor angiogenesis in chemical-induced squamous cell carcinogenesis. Cancer Sci 99: 510-517, 2008.

25. Yang XM, Han HX, Sui F, Dai YM, Chen M and Geng JG: Slit-Robo signaling mediates lymphangiogenesis and promotes tumor lymphatic metastasis. Biochem Biophys Res Commun 396: 571-577, 2010. 